\title{
A New Approach to the Optimization of the CVRP through Genetic Algorithms
}

\author{
Mariano Frutos ${ }^{1}$, Fernando Tohmé ${ }^{2}$ \\ ${ }^{1}$ Department of Engineering, National University of the South, Bahía Blanca, Argentina \\ ${ }^{2}$ Department of Economics, National University of the South, Bahía Blanca, Argentina \\ Email: mfrutos@uns.edu.ar, ftohme@criba.edu.ar
}

Received July 27, 2012; revised August 28, 2012; accepted September 11, 2012

\begin{abstract}
This paper presents a new approach to the analysis of complex distribution problems under capacity constraints. These problems are known in the literature as CVRPs (Capacitated Vehicle Routing Problems). The procedure introduced in this paper optimizes a transformed variant of a CVRP. It starts generating feasible clusters and codifies their ordering. In the next stage the procedure feeds this information into a genetic algorithm for its optimization. This makes the algorithm independent of the constraints and improves its performance. Van Breedam problems have been used to test this technique. While the results obtained are similar to those in other works, the processing times are longer.
\end{abstract}

Keywords: Vehicle Routing Problem; Genetic Algorithms; Modeling; Optimization

\section{Introduction}

The complexity of distribution problems is the reason for the lack of solution techniques able to provide optimal solutions to real cases in a reasonable time [1]. But firms have to make decisions on transportation matters, sometimes in the short run. The economic importance of moving people and goods makes it necessary the development of strategies to generate quick competitive solutions [2,3]. In the literature, these problems are generically known as the Vehicle Routing Problem (VRP) [4], which is NP-Complete combinatorial optimization problem. Its scholarly and practical interest stems from the aforementioned lack of quick solution methods [5]. The main goal of this paper is to introduce a new algorithm, robust, flexible and fast enough to solve different instances of this problem.

The rest of the paper is organized as follows. Firstly, we present some characterizations of the VRP as well as of the original version of the CVRP (Capacitated Vehicle Routing Problem). Then, we introduce an alternative version of the latter and a methodology for its solution. We present later the result of the experiments with this method. Finally we discuss possible further work on the subject.

\section{Definition of CVRP}

In rough terms, the statement of the VRP is: given classes of clients and of storage sites (both scattered over a geographical region) and a fleet of vehicles, determine the minimal cost routes starting and ending in storage sites delivering the goods to the clients. The features of clients, storage sites and vehicles, as well as the operational constraints over routes determine the different variants of the problem. Each client is associated to a demand that has to be covered by a vehicle. This demand is usually understood as an amount of a good that occupies some volume of the vehicle. In crowded area a single vehicle may not be able to satisfy the demands of all the clients living there. The capacity of the vehicle imposes then a bound on the number of clients that it might satisfy at the same time [6,7]. This kind of constraints defines the CVRP (Capacitated Vehicle Routing Problem). The capacity of the vehicle might be in terms of weight or volume. When more than a single kind of products is transported the vehicles could be compartmented carrying each type of parcel in a different subdivision. A distinction can be made between the problems of homogeneous fleets, in which the attributes (capacity, cost, etc.) are the same for all the vehicles, and heterogeneous fleet problems. The latter case raises the possibility of incompatibilities between the capacities of the vehicles and the demands of the clients. If then, each client might be visited only by some of the vehicles. It could also happen that the goods to be transported might not be available in a single storage site and must be collected from several sites. All these depots have to be visited before delivering the goods to the client. While normally each client should be visited once, it could be necessary to satisfy his demand at different moments and by 
different vehicles. Some clients could have some hourly restrictions for the reception of parcels, which are expressed as time windows [8,9]. In such case the problem is known as VRPTW (Vehicle Routing Problem with Time Windows). The vehicles, as well as the products, are usually parked at the storage sites. It is also usually required that the vehicle starts and ends trips from the same storage site, but this restriction can be lifted in some cases. The number of available vehicles could be given or be decision variable [10]. The objective is frequently the minimization of the distances as well as the number of vehicles used while satisfying the demands of the clients [11,12]. Recent studies have allowed the possibility of vehicles traversing different routes [13]. Different presentations of such problems can be found in the literature [14-17], as well as different approaches to solving them [18-23].

In this work we focus on the CVRP. Consider version (1) of the problem. Let $N^{c}$ be the class of client nodes, endowed with a linear order $<, N^{s}$ the set of storage nodes while $N=N^{c} \cup N^{s} . C_{i j}$ the cost (distance) of going from nodes $i$ to $j$ for any pair $(i, j) \in N \times N$. $X_{i j}$ is a binary variable, which is 1 if a vehicle goes from $i$ to $j$ without intermediate stops. Otherwise $X_{i j}=0 . C_{\max }$ is the maximum capacity of a vehicle and $d_{i}$ the amount demanded at a node $i \in N^{c}$. Let $S_{j}$ be the class of nodes in a path that starts and ends at node $j \in N^{s}$. Then, the problem is:

$$
\min \sum_{i \in N} \sum_{j \in N} C_{i j} X_{i j}
$$

s.t.:

1) $\sum_{i \in N^{c} \cap S_{j}} \mathrm{~d}_{i} \leq C_{\max }$,

2) and $\left\{S_{j}\right\}_{j \in N^{s}}$ such that $\left(S_{i} \cap S_{j}\right) \cap N^{c}=\varnothing$

In words: minimize the total distance traversed by all vehicles, satisfying the demands on every route for a partition of client nodes in clusters, each one covered by a single vehicle. Notice that constraint 2) makes the problem more complex than a linear programming one.

Version (2) is as follows: let $M=\left\{S_{j}\right\}_{j \in N^{s}}$ for some allocation of routes, $c_{m}=\sum_{(i, j) \in m} C_{i j}$ the sum of distances over route $m, X_{m}$ is a binary variable that is 1 if route $m$ is used and 0 otherwise and $e_{i m}$ also a binary variable ( 1 if $i \in N^{c}$ belongs to route $m$ and 0 otherwise). Then, the problem is:

$$
\min \sum_{m \in M} c_{m} X_{m}
$$

s.t.:

3) and for each $i, \quad \sum_{m \in M} e_{i m} X_{\mathrm{m}}=1$,

That is (2) is analogous to (1) under constraint 1 ) with constraint 2) already satisfied.

\section{Methodology}

An algorithm solving (2) proceeds in two stages. The first one generates feasible clusters, classifying and ordering them. The second stage solves the minimization problem by means of a Genetic Algorithm [24].

\subsection{First Stage}

The algorithm generates the clusters that will be elements of $\left\{S_{j}\right\}_{j \in N^{S}}$. We take each $i \in N^{c}$ and consider all the clusters $S_{j}$ such that $i \in S_{j}$. The inductive construction starts with $i=1$ (the lowest in the $<$ order) building all the clusters of client nodes that include 1 , satisfying condition 1). Then, if all possible clusters up to $i=k$ have been obtained, consider $i=k+1$ (where, of course, $k<k$ +1 ). All routes starting and ending in a node of $N^{s}$ that go through client nodes in $N^{c}-\{1, \cdots, k\}$, including node $k+1$, satisfying condition 1 ), are constructed.

For each $i \in N^{c}$ its class of clusters, denoted $S^{i}$, is endowed with a weak order $\angle_{i}$ (reflexive, antisymmetric and transitive, i.e. allowing ties) according to the number of clients in each cluster. Among clusters covering the same number of client nodes, say $\{1, \cdots, i, \cdots, k\}$ and $\{1, \cdots, i, \cdots, l\}$, if $k<l$ then $\{1, \cdots, i, \cdots, k\} \angle_{i}$ $\{1, \cdots, i, \cdots, l\}$. The highest rank in $\angle_{i}$ corresponds to routes covering $i$ as well as the largest possible number of other client nodes in the upper level of order $<$. As an example, consider the case of 10 client nodes demanding 50 units and a vehicle capacity of 100 units. Table 1 shows the distances among nodes, being 0 the storage site from which departs the vehicle. Table 2 shows the result of the first stage of the procedure. Notice that, since the capacity of the vehicle is 100 and each client node demands 50, a route that clusters some clients together can only consist of two of them. So, for instance the group corresponding to node 9 includes only two possible clusters, $\{9\}$ and $\{9,10\}$, because all other nodes have already been assigned to other clusters. Cluster $\{9,10\}$ is of a higher order than $\{9\}$ because it includes two clients instead of just one node.

\subsection{Second Stage}

At this point a Genetic Algorithm [24] is applied, designed for its use in this problem. An individual is codified in a chromosome constructed in such way as to satisfy conditions 2) and 3) and therefore are feasible under problem (2). The coding operates on a lexicographic basis. Each $i \in N^{c}$ is given a number, starting with $i=1$, and following the process according to order $<$. So, take all the clusters ordered according to $\angle_{1}$. This order can be represented by the linear numerical order $1, \cdots,\left|S^{1}\right|$. So, if the $k$-th cluster in the ordering is chosen, the corresponding gene in the chromosome is $k$. For $i=j$, the 
Table 1. Distances-Case: 10 nodes / Demand per node: 50 units / Vehicle capacity: 100 units.

\begin{tabular}{|c|c|c|c|c|c|c|c|c|c|c|c|}
\hline Nodes & 0 & 1 & 2 & 3 & 4 & 5 & 6 & 7 & 8 & 9 & 10 \\
\hline 0 & 0.00 & 5.00 & 14.14 & 7.07 & 11.18 & 15.81 & 14.14 & 5.00 & 11.18 & 11.18 & 18.03 \\
\hline 1 & 5.00 & 0.00 & 11.18 & 11.18 & 15.81 & 20.62 & 18.03 & 10.00 & 7.07 & 10.00 & 22.36 \\
\hline 2 & 14.14 & 11.18 & 0.00 & 15.81 & 25.00 & 25.50 & 20.00 & 18.03 & 5.00 & 20.62 & 32.02 \\
\hline 3 & 7.07 & 11.18 & 15.81 & 0.00 & 11.18 & 10.00 & 7.07 & 5.00 & 15.00 & 18.03 & 18.03 \\
\hline 4 & 11.18 & 15.81 & 25.00 & 11.18 & 0.00 & 11.18 & 15.00 & 7.07 & 22.36 & 15.81 & 7.07 \\
\hline 6 & 14.14 & 18.03 & 20.00 & 7.07 & 15.00 & 7.07 & 0.00 & 11.18 & 20.62 & 25.00 & 20.62 \\
\hline 7 & 5.00 & 10.00 & 18.03 & 5.00 & 7.07 & 11.18 & 11.18 & 0.00 & 15.81 & 14.14 & 14.14 \\
\hline 8 & 11.18 & 7.07 & 5.00 & 15.00 & 22.36 & 25.00 & 20.62 & 15.81 & 0.00 & 15.81 & 29.15 \\
\hline 9 & 11.18 & 10.00 & 20.62 & 18.03 & 15.81 & 25.00 & 25.00 & 14.14 & 15.81 & 0.00 & 20.00 \\
\hline 10 & 18.03 & 22.36 & 32.02 & 18.03 & 7.07 & 15.00 & 20.62 & 14.14 & 29.15 & 20.00 & 0.00 \\
\hline
\end{tabular}

Table 2. Clusters-Case: 10 nodes / Demand per node: 50 units / Vehicle capacity: 100 units.

\begin{tabular}{cccccccccccc}
\hline Nodes & & & \multicolumn{7}{c}{ Clusters } \\
\hline $\mathbf{0}$ & - & - & - & - & - & - & - & - & - \\
$\mathbf{1}$ & 1 & 1.2 & 1.3 & 1.4 & 1.5 & 1.6 & 1.7 & 1.8 & 1.9 & 1.10 \\
$\mathbf{2}$ & 2 & 2.3 & 2.4 & 2.5 & 2.6 & 2.7 & 2.8 & 2.9 & 2.10 & - \\
$\mathbf{3}$ & 3 & 3.4 & 3.5 & 3.6 & 3.7 & 3.8 & 3.9 & 3.10 & - & - \\
$\mathbf{4}$ & 4 & 4.5 & 4.6 & 4.7 & 4.8 & 4.9 & 4.10 & - & - & - \\
$\mathbf{5}$ & 5 & 5.6 & 5.7 & 5.8 & 5.9 & 5.10 & - & - & - & - \\
$\mathbf{6}$ & 6 & 6.7 & 6.8 & 6.9 & 6.10 & - & - & - & - & - \\
$\mathbf{7}$ & 7 & 7.8 & 7.9 & 7.10 & - & - & - & - & - & - \\
$\mathbf{8}$ & 8 & 8.9 & 8.10 & - & - & - & - & - & - & - \\
$\mathbf{9}$ & 9 & 9.10 & - & - & - & - & - & - & - & - \\
$\mathbf{1 0}$ & 10 & - & - & - & - & - & - & - & - \\
\hline
\end{tabular}

order $\angle_{j}$ is applied to a subset of $S^{j}$ obtained by eliminating ("filtered out") all the clusters that include nodes already present in clusters corresponding to genes $i=$ $1, \cdots, j-1$. The new ordering is denoted $\angle i^{\prime}$. Then the $\mathrm{j}$-th gene in the chromosome is the rank in $\angle i^{\prime}$ of the cluster that is chosen. If for a given $i$ the domain obtained is $\varnothing$ (because all possible nodes belong to clusters corresponding to genes 1 to $i-1$ ), any number between 1 and $\left|S^{i}\right|$ can be assigned to the $i$-th gene. In this way, a chromosome is defined, each corresponding to a feasible solution to problem (2). The Genetic Algorithm seeks to find the optimal one.

We can see some examples of how chromosomes encode clusters in Table 3, (3-3-2-1-4-2-3-2-1-1) and Table 4 (5-2-1-5-1-4-2-2-1-1): all routes start and end at node 0 and go through two client nodes; in gray we see the filtered out clusters and in red the selection. Consider for instance the second gene in Table 3: it has to discard (written in gray) all routes that go through node 3 , since it has already been assigned to the first gene. Number 3 assigned to this gene indicate that the route selected is the third in the list of non-filtered routes. On the other hand, a number assigned to a gene with a row with all routes filtered out does not have any effect. The initial population consists of individuals whose chromosomes are constituted by genes selected at random. Once established a chromosome, it is decoded and evaluated. This evaluation is performed according to problem (2). For the computation of each cluster we use Lin-Kerninghan's algorithm that yields the minimal distance among its nodes. The following operators have been chosen in the light of some previous evaluations. The cross-over operator is the two-point one (Table 5, Parent 1: 3-32-_-_-_-_-2-1-1, Parent 2: _-_-_-5-1-4-2-_-_-_, Offspring: 3-3-2-5-1-4-2-2-1-1) and the mutation operator alters gene values at $10 \%$ of the chromosome (Mutation (Table 6, Offspring*: 3-3-2-5-1-2-2-2-1-1)). Finally, the selection is by ranking.

The Genetic Algorithm runs until the solution gets stabilized. These tests were implemented with a $\mathrm{C}^{++}$program running on a $3.00 \mathrm{GHZ}$ CPU with a 2.00 GB RAM. The parameters of the experiments were as follows. The size of the population: 100; the cross-over probability: 0.70 and the probability of mutation: 0.01 .

Before running the algorithm over problems in the literature we experimented over this example. Figure 1 shows the nodes and their proportional distances in a two-dimensional framework. Figures 2 to 7 show the 
Table 3. Example of a chromosome (3-3-2-1-4-2-3-2-1-1) that will act as parent 1 in the cross-over of Table 5.

\begin{tabular}{ccccccccccccc}
\hline Chr. & Nodes & \multicolumn{10}{c}{ Clusters } \\
\hline $\mathbf{3}$ & $\mathbf{1}$ & $0-1-0$ & $0-1-2-0$ & $0-1-3-0$ & $0-1-4-0$ & $0-1-5-0$ & $0-1-6-0$ & $0-1-7-0$ & $0-1-8-0$ & $0-1-9-0$ & $0-1-10-0$ \\
$\mathbf{3}$ & $\mathbf{2}$ & $0-2-0$ & $0-2-3-0$ & $0-2-4-0$ & $0-2-5-0$ & $0-2-6-0$ & $0-2-7-0$ & $0-2-8-0$ & $0-2-9-0$ & $0-2-10-0$ & - \\
$\mathbf{2}$ & $\mathbf{3}$ & $0-3-0$ & $0-3-4-0$ & $0-3-5-0$ & $0-3-6-0$ & $0-3-7-0$ & $0-3-8-0$ & $0-3-9-0$ & $0-3-10-0$ & - \\
$\mathbf{1}$ & $\mathbf{4}$ & $0-4-0$ & $0-4-5-0$ & $0-4-6-0$ & $0-4-7-0$ & $0-4-8-0$ & $0-4-9-0$ & $0-4-10-0$ & - & - \\
$\mathbf{4}$ & $\mathbf{5}$ & $0-5-0$ & $0-5-6-0$ & $0-5-7-0$ & $0-5-8-0$ & $0-5-9-0$ & $0-5-10-0$ & - & - & - & - \\
$\mathbf{2}$ & $\mathbf{6}$ & $0-6-0$ & $0-6-7-0$ & $0-6-8-0$ & $0-6-9-0$ & $0-6-10-0$ & - & - & - & - & - \\
$\mathbf{3}$ & $\mathbf{7}$ & $0-7-0$ & $0-7-8-0$ & $0-7-9-0$ & $0-7-10-0$ & - & - & - & - & - & - \\
$\mathbf{2}$ & $\mathbf{8}$ & $0-8-0$ & $0-8-9-0$ & $0-8-10-0$ & - & - & - & - & - & - & - \\
$\mathbf{1}$ & $\mathbf{9}$ & $0-9-0$ & $0-9-10-0$ & - & - & - & - & - & - & - & - \\
$\mathbf{1}$ & $\mathbf{1 0}$ & $0-10-0$ & - & - & - & - & - & - & - & - & - \\
\hline
\end{tabular}

Table 4. Example of a chromosome (5-2-1-5-1-4-2-2-1-1) that will act as parent 2 in the cross-over of Table 5.

\begin{tabular}{|c|c|c|c|c|c|c|c|c|c|c|c|}
\hline \multirow{2}{*}{$\begin{array}{c}\text { Chr. } \\
5\end{array}$} & \multirow{2}{*}{$\begin{array}{c}\text { Nodes } \\
1\end{array}$} & \multicolumn{10}{|c|}{ Clusters } \\
\hline & & $0-1-0$ & $0-1-2-0$ & $0-1-3-0$ & $0-1-4-0$ & $0-1-5-0$ & $0-1-6-0$ & $0-1-7-0$ & $0-1-8-0$ & $0-1-9-0$ & $0-1-10-0$ \\
\hline 2 & 2 & $0-2-0$ & $0-2-3-0$ & $0-2-4-0$ & $0-2-5-0$ & $0-2-6-0$ & $0-2-7-0$ & $0-2-8-0$ & $0-2-9-0$ & $0-2-10-0$ & - \\
\hline 5 & 4 & $0-4-0$ & $0-4-5-0$ & $0-4-6-0$ & $0-4-7-0$ & $0-4-8-0$ & $0-4-9-0$ & $0-4-10-0$ & - & - & - \\
\hline 1 & 5 & $0-5-0$ & $0-5-6-0$ & $0-5-7-0$ & $0-5-8-0$ & $0-5-9-0$ & $0-5-10-0$ & - & - & - & - \\
\hline 4 & 6 & $0-6-0$ & $0-6-7-0$ & $0-6-8-0$ & $0-6-9-0$ & $0-6-10-0$ & - & - & - & - & - \\
\hline 2 & 7 & $0-7-0$ & $0-7-8-0$ & $0-7-9-0$ & $0-7-10-0$ & - & - & - & - & - & - \\
\hline 2 & 8 & $0-8-0$ & 0-8-9-0 & $0-8-10-0$ & - & - & - & - & - & - & - \\
\hline 1 & 9 & $0-9-0$ & $0-9-10-0$ & - & - & - & - & - & - & - & - \\
\hline 1 & 10 & $0-10-0$ & - & - & - & - & - & - & - & - & - \\
\hline
\end{tabular}

Table 5. Cross-over (offspring: 3-3-2-5-1-4-2-2-1-1).

\begin{tabular}{|c|c|c|c|c|c|c|c|c|c|c|c|}
\hline \multirow{2}{*}{$\begin{array}{c}\text { Chr. } \\
3\end{array}$} & \multirow{2}{*}{$\begin{array}{c}\text { Nodes } \\
1\end{array}$} & \multicolumn{10}{|c|}{ Clusters } \\
\hline & & $0-1-0$ & $0-1-2-0$ & $0-1-3-0$ & $0-1-4-0$ & $0-1-5-0$ & $0-1-6-0$ & $0-1-7-0$ & $0-1-8-0$ & $0-1-9-0$ & $0-1-10-0$ \\
\hline 3 & 2 & $0-2-0$ & $0-2-3-0$ & $0-2-4-0$ & $0-2-5-0$ & $0-2-6-0$ & $0-2-7-0$ & $0-2-8-0$ & $0-2-9-0$ & $0-2-10-0$ & - \\
\hline 5 & 4 & $0-4-0$ & $0-4-5-0$ & $0-4-6-0$ & $0-4-7-0$ & $0-4-8-0$ & $0-4-9-0$ & $0-4-10-0$ & - & - & - \\
\hline 1 & 5 & $0-5-0$ & $0-5-6-0$ & $0-5-7-0$ & $0-5-8-0$ & $0-5-9-0$ & $0-5-10-0$ & - & - & - & - \\
\hline 4 & 6 & $0-6-0$ & $0-6-7-0$ & $0-6-8-0$ & $0-6-9-0$ & $0-6-10-0$ & - & - & - & - & - \\
\hline 2 & 7 & $0-7-0$ & $0-7-8-0$ & $0-7-9-0$ & $0-7-10-0$ & - & - & - & - & - & - \\
\hline 2 & 8 & $0-8-0$ & $0-8-9-0$ & $0-8-10-0$ & - & - & - & - & - & - & - \\
\hline 1 & 9 & $0-9-0$ & $0-9-10-0$ & - & - & - & - & - & - & - & - \\
\hline 1 & 10 & $0-10-0$ & - & - & - & - & - & - & - & - & - \\
\hline
\end{tabular}

Table 6. Mutation (offspring*: 3-3-2-5-1-4-2-2-1-1).

\begin{tabular}{|c|c|c|c|c|c|c|c|c|c|c|c|}
\hline \multirow{2}{*}{$\begin{array}{c}\text { Chr. } \\
3\end{array}$} & \multirow{2}{*}{$\begin{array}{c}\text { Nodes } \\
1\end{array}$} & \multicolumn{10}{|c|}{ Clusters } \\
\hline & & $0-1-0$ & $0-1-2-0$ & $0-1-3-0$ & $0-1-4-0$ & $0-1-5-0$ & $0-1-6-0$ & $0-1-7-0$ & $0-1-8-0$ & $0-1-9-0$ & $0-1-10-0$ \\
\hline 3 & 2 & $0-2-0$ & $0-2-3-0$ & $0-2-4-0$ & $0-2-5-0$ & $0-2-6-0$ & $0-2-7-0$ & $0-2-8-0$ & $0-2-9-0$ & $0-2-10-0$ & - \\
\hline 2 & 3 & $0-3-0$ & $0-3-4-0$ & $0-3-5-0$ & $0-3-6-0$ & $0-3-7-0$ & $0-3-8-0$ & $0-3-9-0$ & $0-3-10-0$ & - & - \\
\hline 5 & 4 & $0-4-0$ & $0-4-5-0$ & $0-4-6-0$ & $0-4-7-0$ & $0-4-8-0$ & $0-4-9-0$ & $0-4-10-0$ & - & - & - \\
\hline 1 & 5 & $0-5-0$ & $0-5-6-0$ & $0-5-7-0$ & $0-5-8-0$ & $0-5-9-0$ & $0-5-10-0$ & - & - & - & - \\
\hline 2 & 7 & $0-7-0$ & 0-7-8-0 & $0-7-9-0$ & $0-7-10-0$ & - & - & - & - & - & - \\
\hline 2 & 8 & $0-8-0$ & $0-8-9-0$ & $0-8-10-0$ & - & - & - & - & - & - & - \\
\hline 1 & 9 & $0-9-0$ & $0-9-10-0$ & - & - & - & - & - & - & - & - \\
\hline 1 & 10 & $0-10-0$ & - & - & - & - & - & - & - & - & - \\
\hline
\end{tabular}




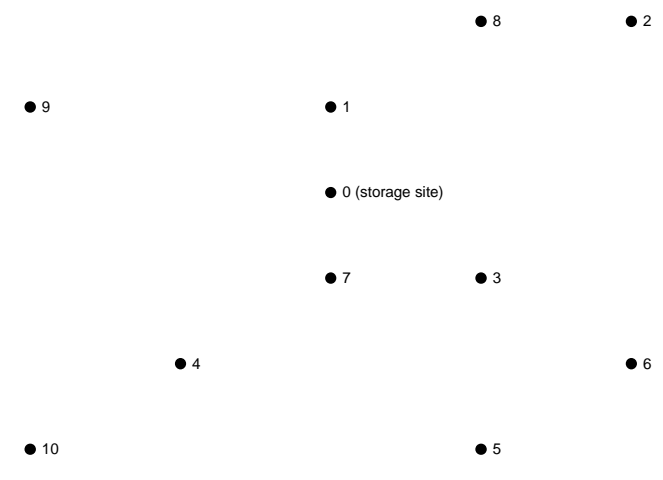

Figure 1. Topology of the problem.

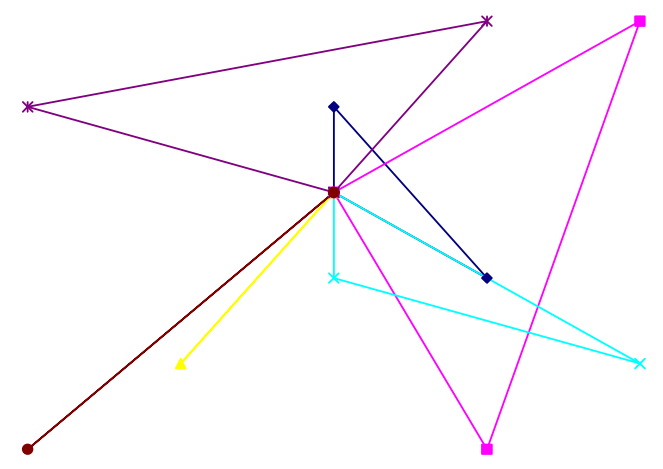

Figure 2. Generation 10, distance: 205.61.

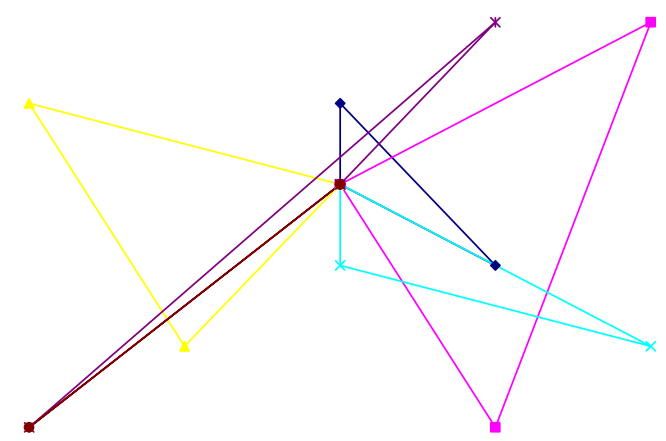

Figure 3. Generation 20, distance: 205.56 .

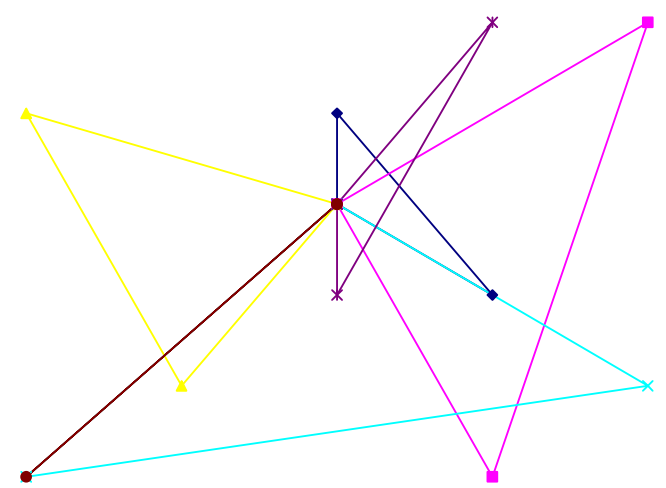

Figure 4. Generation 45, distance: 201.65.

routes generated in several generations and the minimal total distance covered by the selected routes.

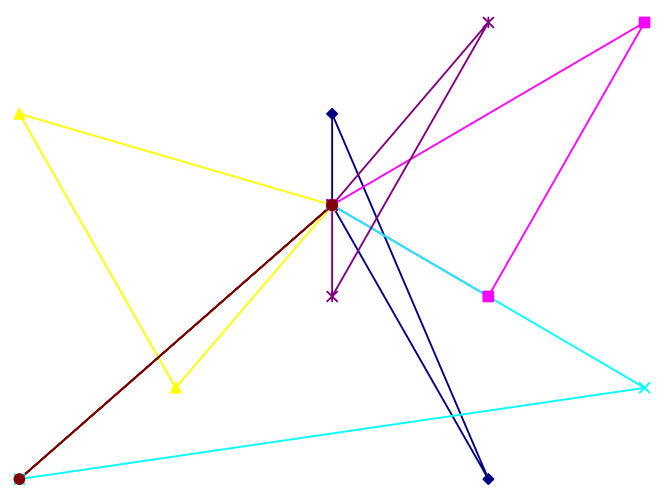

Figure 5. Generation 75, distance: 201.40.

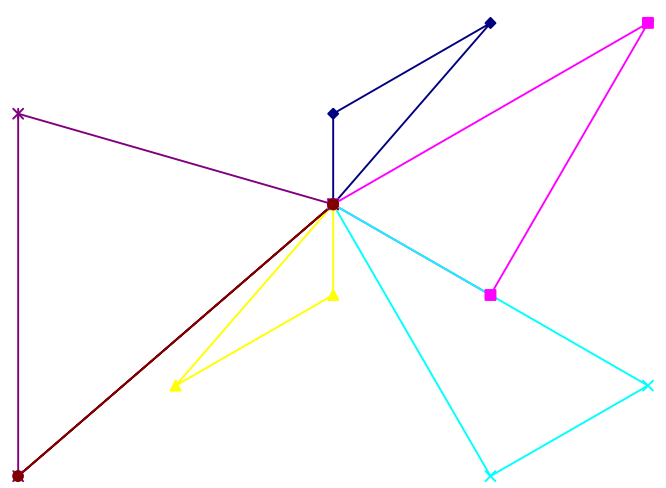

Figure 6. Generation 100, distance: 169.76.

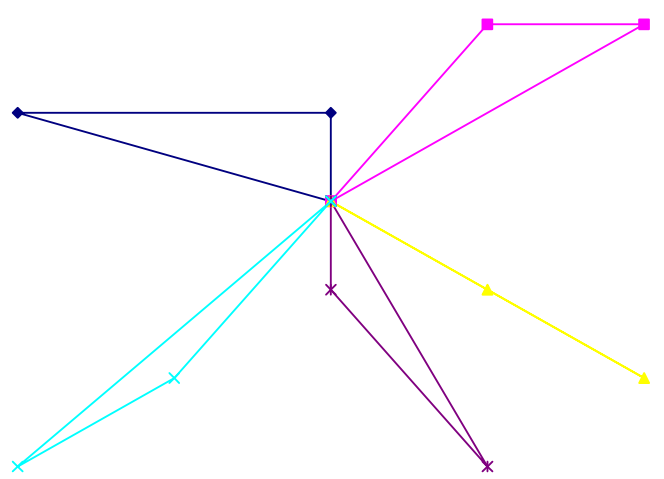

Figure 7. Generation 150, distance: 153.06.

\section{Experiments and Results}

Van Breedam [25] presents a large number of instances of features and values. He suggested also a reduced set of representative cases to be solved by different heuristics. Some of them involve 100 nodes with a total demand of 1000 units. We follow here this convention and use the author's nomenclature for the cases with the same capacity of vehicles. The problems tested range from Bre- 1 to Bre-15. From Bre-1 to Bre- 6 only the capacity of vehicles is restricted. The demand on each client node is 10 units, while the vehicle capacity is 100 units in Bre-1 and Bre-2, 50 units in Bre-3 and Bre-4, while it is of 200 
Table 7. Results for the problems of Van Breedam [25,26].

\begin{tabular}{|c|c|c|c|c|c|c|c|}
\hline \multirow{2}{*}{ Instance } & \multirow{2}{*}{ Best Known Solution } & \multicolumn{6}{|c|}{ Best Solution Found } \\
\hline & & Best Solution & Evaluations & Success (\%) & Average Solution & $\Delta(\%)$ & Average Time (min) \\
\hline Bre-1 & 1106.00 & 1157.00 & $395347.12 \pm 125177.89$ & 42.00 & $1169.02 \pm 14.76$ & -4.61 & 25.16 \\
\hline Bre-2 & 1506.00 & 1587.00 & $374623.02 \pm 25544.61$ & 18.00 & $1595.75 \pm 16.91$ & -5.38 & 24.11 \\
\hline Bre-3 & 1751.00 & 1751.00 & $121640.67 \pm 25684.27$ & 100.00 & $1751.00 \pm 0.00$ & 0.00 & 11.20 \\
\hline Bre-4 & 1470.00 & 1470.00 & $287936.32 \pm 14739.13$ & 100.00 & $1470.00 \pm 0.00$ & 0.00 & 19.68 \\
\hline Bre-5 & 950.00 & 985.00 & $326733.84 \pm 112846.01$ & 76.00 & $994.12 \pm 11.49$ & -3.68 & 21.66 \\
\hline Bre-6 & 969.00 & 979.00 & $523967.47 \pm 162502.07$ & 62.00 & $982.43 \pm 6.59$ & -1.03 & 31.72 \\
\hline Bre-9 & 1690.00 & 1790.00 & $657281.22 \pm 21838.39$ & 17.00 & $1797.93 \pm 8.03$ & -5.92 & 38.52 \\
\hline Bre-10 & 1026.00 & 1026.00 & $871531.96 \pm 326830.70$ & 52.00 & $1035.85 \pm 9.42$ & 0.00 & 49.45 \\
\hline Bre-11 & 1128.00 & 1187.00 & $1342635.29 \pm 543765.65$ & 100.00 & $1187.00 \pm 0.00$ & -5.23 & 73.47 \\
\hline
\end{tabular}

units in Bre-5 and Bre-6. Problems Bre-7 and Bre-8 have not been considered because they involve both deliveries and pickups. In most of these problems a single type of good is assumed. Exceptions are problems Bre-9 to Bre-11, in which demands are heterogeneous. Finally Bre-12 to Bre-15 are problems involving time windows, and therefore are not included in our experiments. Table 7 presents the results of our tests. The best known solution, drawn from $[25,26]$, is presented as well as information on the solutions under our procedure (best solution, average evaluation, success, mean solution, average running times and percentage of difference with the best known solution). With respect to the processing time, some instances took only minutes while others required hours, although in average the best solutions took not much longer than an hour to be found.

\section{Conclusion}

We presented a new algorithm for the CVRP (Capacitated Vehicle Routing Problem), distinguishing between the standard and its alternative formulation. The details of the procedure were described and the comparison with results in the literature has been presented. While the outcomes are similar to them, their running times are not. In experiments not discussed here we noticed that problems that exceed 100 nodes the processing time is much longer, due to the demands of the first stage of the algorithm. We plan to add further search filters at the optimization stage as well as to try with other meta-heuristics to run on the alternative model.

\section{Acknowledgements}

This work has been supported by several funding sources. The authors are in particular thankful to the National Research Council of Argentina (CONICET) as well as to the Universidad Nacional del Sur project PGI 24/J056.

\section{REFERENCES}

[1] D. Ambrosino and A. Sciomachen, "A Food Distribution Network Problem: A Case Study,” IMA Journal of Management Mathematics, Vol. 18, No. 1, 2007, pp. 33-53. doi:10.1093/imaman/dpl012

[2] L. D. Bodin and B. L. Golden, "Classification in Vehicle Routing and Scheduling,” Networks, Vol. 11. No. 2, 1981, pp. 97-108. doi:10.1002/net.3230110204

[3] S. N. Kumar and R. Panneerselvam, "A Survey on the Vehicle Routing Problem and Its Variants," Intelligent Information Management, Vol. 4, No. 3, 2012, pp. 66-74. doi:10.4236/iim.2012.43010

[4] G. Laporte and I. H. Osman, "Routing Problems: A Bibliography," Annals of Operations Research, Vol. 61, No. 1, 1995, pp. 227-262. doi:10.1007/BF02098290

[5] E. Mota, V. Campos and A. Corbéran, “A New Metaheuristic for the Vehicle Routing Problem with Split Demands," In: J. van Hemert and C. Cotta, Eds., Evolutionary Computation in Combinatorial Optimization, Lecture Notes in Computer Science Vol. 4446, Springer-Verlag, Berlin, 2007, pp. 121-129. doi:10.1007/978-3-540-71615-0 11

[6] R. Tavakkoli-Moghaddam, N. Safaei, M. Kah and M. Rabbani, "A New Capacitated Vehicle Routing Problem with Split Service for Minimizing Fleet Cost by Simulated Annealing," Journal of the Franklin Institute, Vol. 344, No. 5, 2007, pp. 406-425. doi:10.1016/j.jfranklin.2005.12.002

[7] J. M. Belenguer, E. Benavent, N. Labadi, C. Prins and M. Reghioui, "Split Delivery Capacitated Arc-Routing Problem: Lower Bound and Metaheuristic,” Transportation Science, Vol. 44, No. 2, 2010, pp. 206-220. doi:10.1287/trsc.1090.0305

[8] S. R. Thangiah, A. Fergany and S. Awan, "Real-Time Split-Delivery Pickup and Delivery Time Window Problems with Transfers," Central European Journal of Operations Research, Vol. 15, No. 4, 2007, pp. 329-349. doi:10.1007/s10100-007-0035-x

[9] M. Desrochers, J. Desrosiers and M. M. Solomon, “A 
New Optimization Algorithm for the Vehicle Routing Problem with Time Windows," Operations Research, Vol. 40, No. 2, 1002, pp. 342-354. doi:10.1287/opre.40.2.342

[10] C. G. Lee, M. A. Epelman, C. White and Y. A. Bozer, “A Shortest Path Approach to the Multiple-Vehicle Routing Problem with Split Pick-Ups,” Transportation Research, Vol. 40, No. 4, 2006, pp. 265-284. doi:10.1016/j.trb.2004.11.004

[11] P. Belfiore and H. T. I. Yoshizaki, "Scatter Search for a Real-Life Heterogeneous Fleet Vehicle Routing Problem with Time Windows and Split Deliveries in Brazil," European Journal of Operational Research, Vol. 199, No. 3, 2009, pp. 750-758. doi:10.1016/j.ejor.2008.08.003

[12] J. H. Wilck IV and T. M. Cavalier, "A Construction Heuristic for the Split Delivery Vehicle Routing," American Journal of Operations Research, Vol. 2, No. 2, 2012, pp. 153-162. doi:10.4236/ajor.2012.22018

[13] Y. Nagao and H. Nagamochi, “A DP-Based Heuristic Algorithm for the Discrete Split Delivery Vehicle Routing Problem," Journal of Advanced Mechanical Design, Systems, and Manufacturing, Vol. 1, No. 2, 2007, pp. 217-226. doi:10.1299/jamdsm.1.217

[14] Y. Yu, H. Chen and F. Chu, "A New Model and Hybrid Approach for Large Scale Inventory Routing Problems," European Journal of Operational Research, Vol. 189, No. 3, 2008, pp. 1022-1040. doi:10.1016/j.ejor.2007.02.061

[15] M. A. Nowak, O. Ergun and C. C. White, "An Empirical Study on the Benefit of Split Loads with the Pickup and Delivery Problem,” European Journal of Operational Research, Vol. 198, No. 3, 2009, pp. 734-740. doi:10.1016/j.ejor.2008.09.041

[16] Z. Shen, M. M. Dessouky and F. Ordóñez, “A Two-Stage Vehicle Routing Model for Large-Scale Bioterrorism Emergencies," Networks, Vol. 54, No. 4, 2009, pp. 255-269. doi:10.1002/net.20337

[17] L. Moreno, M. P. de Aragao and E. Uchoa, "Improved Lower Bounds for the Split Delivery Vehicle Routing Problem,” Operations Research Letters, Vol. 38, No. 4, 2010, pp. 302-306. doi:10.1016/j.orl.2010.04.008

[18] M.-C. Bolduc, G. Laporte, J. Renaud and F. F. Boctor, “A Tabu Search Heuristic for the Split Delivery Vehicle Rout- ing Problem with Production and Demand Calendars," European Journal of Operational Research, Vol. 202, No. 1, 2010, pp. 122-130. doi:10.1016/j.ejor.2009.05.008

[19] U. Derigs, B. Li and U. Vogel, "Local Search-Based Metaheuristics for the Split Delivery Vehicle Routing Problem," Journal of the Operational Research Society, Vol. 61, No. 9, 2009, pp. 1356-1364. doi:10.1057/jors.2009.100

[20] M. Jin, K. Liu and R. O. Bowden, “A Two-Stage Algorithm with Valid Inequalities for the Split Delivery Vehicle Routing Problem,” International Journal of Production Economics, Vol. 105, No. 1, 2007, pp. 228-242. doi:10.1016/j.ijpe.2006.04.014

[21] R. E. Aleman and R. R. Hill, “A Tabu Search with Vocabulary Building Approach for the Vehicle Routing Problem with Split Demands,” International Journal of Metaheuristics, Vol. 1, No. 1, 2010, pp. 55-80. doi:10.1504/IJMHEUR.2010.033123

[22] R. E. Aleman, X. Zhang and R. R. Hill, “An Adaptive Memory Algorithm for the Split Delivery Vehicle Routing Problem,” Journal of Heuristics, Vol. 16, No. 3, 2010, pp. 441-473. doi:10.1007/s10732-008-9101-3

[23] S. Basu, "Tabu Search Implementation on Traveling Salesman Problem and Its Variations: A Literature Survey," American Journal of Operations Research, Vol. 2, No. 2, 2012, pp. 163-173. doi:10.4236/ajor.2012.22019

[24] D. Goldberg, "Genetic Algorithms in Search, Optimization, and Machine Learning," Addison-Wesley Longman Publishing Co., Inc., Boston, 1989.

[25] A. Van Breedam, "An Analysis of the Behavior of Heuristics for the Vehicle Routing Problem for a Selection of Problems with Vehicle Related, Customer-Related, and Time-Related Constraints,” Ph.D. Thesis, University of Antwerp-RUCA, Antwerpen, 1994.

[26] E. Alba and B. Dorronsoro, "A Hybrid Cellular Genetic Algorithm for the Capacitated Vehicle Routing Problem," In: Engineering Evolutionary Intelligent Systems, Chapter 13, Studies in Computational Intelligence, SpringerVerlag, Heidelberg, 2008, pp. 379-422. doi:10.1007/978-3-540-75396-4_14 